\title{
The Inverse Honeycomb Structures in Numerical Modeling and Experiment
}

\author{
Malgorzata JOHN*, Antoni JOHN**, Wojciech SKARKA*** \\ *Silesian University of Technology, Faculty of Mechanical Engineering, Institute of Fundamentals of Machinery Design, \\ Konarskiego 18A, 44-100 Gliwice, Poland, E-mail: malgorzata.john@polsl.pl \\ **Silesian University of Technology, Faculty of Mechanical Engineering, Institute of Computational Mechanics and \\ Engineering, Konarskiego 18A, 44-100 Gliwice, Poland, E-mail: antoni.john@polsl.pl \\ ***Silesian University of Technology, Faculty of Mechanical Engineering, Institute of Fundamentals of Machinery \\ Design, Konarskiego 18A, 44-100 Gliwice, Poland, E-mail: wojciech.skarka@polsl.pl
}

cross $^{\text {ref }}$ http://dx.doi.org/10.5755/j01.mech.24.3.21066

\section{Introduction}

Sandwich structure consisting of a core and cladding has been used in various industries such as aerospace, shipbuilding, automotive industry. The core layer in most cases is thicker than the outer layer $[1,2]$. Such a structure greatly varies its properties of conventional construction, among others, due to the anisotropy of stiffness. Both materials are in themselves strong and rigid, but the composite formed from them has much rigidity and strength. It has several important features that are important in designing and developing in these industries. This structure is characterized by a relatively low weight in relation to mechanical properties [3]. By modifying the core and cladding may be some influence on these properties. An interesting feature is the ability to absorb and absorption characteristics in a wide range of frequencies from infrasound, vibration or mechanical, to the acoustic vibration frequencies or tones. The structure also showed resistance to corrosion in the marine environment and heat resistance. In the aerospace industry and shipbuilding proved significant weight construction. The automotive industry has focused mainly on the properties described absorbent structure. It turns out to be useful for building security systems.

Conducted a review of literature concerning the strength tests carried out on sandwich structures with different types of core e.g. by the cells of the honeycomb. If you try to three-point bending it is most often carry up to break the sample in all layers. Scientists from China con-ducted the test of endurance on the structure of the corrugated core type [4]. During the test was interrupted so-called external coatings. skin. The core during operation forces underwent deformation, but not ripped up. After unloading the inner layer of the sample returned to a state similar to the one at the start of the study.

\section{Mechanics of cracking}

Mechanics of cracking is a field dealing with the description of phenomena occurring in the material in the vicinity of a sharp notch, i.e. at the apex of the fracture. Here the problem appears during experiment. Initial crack started in opposite side of acting force and propagates in different way. That issue has complicated in the initial models of inverse honeycomb structures without transition between cells. These phenomena can be reduced to three main issues $[5,6]$ :
- concentration of stresses,

- stress intensity

- material resistance to crack initiation at the apex of the fracture.

Stress concentration factor is defined as the ratio of stress induced in the bottom of the notch $\sigma_{\max }$ to the nominal stress:

$$
C=\frac{\sigma_{\max }}{\sigma}
$$

If one considers the gap in the form of an elliptical hole with a large axis $2 \mathrm{a}$ and a small axis $2 \mathrm{~b}$ (Fig. 1), then the stress concentration factor is:

$$
C=1+2 \frac{a}{b} \text { or } C=1+\frac{a}{\rho}
$$

where: $\rho$ is the radius of curvature at the ends of the ellipse.

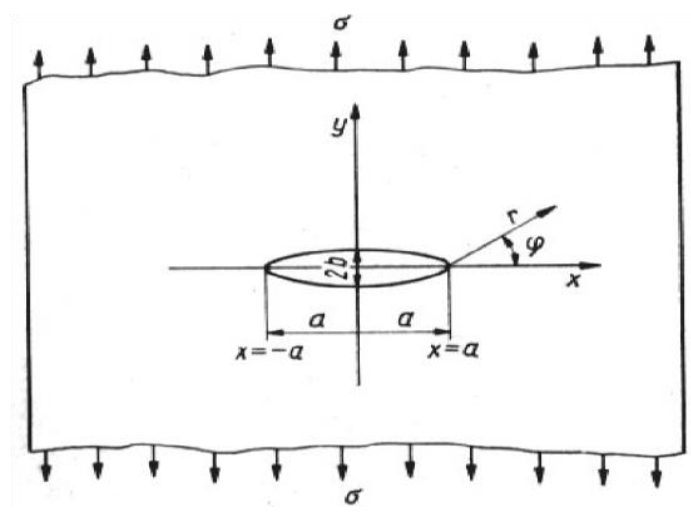

Fig. 1 The development of a gap in the material of the board with an internal notch in the shape of an ellipse - the state before the development of the crack [6]

If you take extreme values for the dimensions of the elliptical axes, you will get:

- for a circular hole $(a=b): C=3$,

- for infinitesimal curvature radius: $C \rightarrow \infty$.

In the latter case, when the coefficient $\mathrm{C}$ tends to infinity, determining it loses its meaning. That is why the so-called coefficient of stress intensity, which retains the finite value, even when $C \rightarrow \infty$. This coefficient is denoted by 
$K$. For the uniaxial tensioned axis along the axis perpendicular to the fracture surface (as in Fig. 1) is defined as the limit to which the product of normal stress tends to the crack surface and the square root from the distance measured from the face of the slot multiplied by factor $2 \pi[7]$.

The stress intensity factor depends on the size of the gap and the applied load, in other words - on the gapexternal load configuration and describes the stress field (as well as the displacement field) in the immediate vicinity of the slot front. In conditions where the nominal stress $\sigma$ for a given gap length a reaches the value at which the development of gap length development (crack initiation) takes place, the stress intensity factor reaches the critical value and is referred to as the $K_{\mathrm{C}}$ symbol. It is particularly important for the testing of materials, as it is a measure of the material's resistance to cracking [6-8].

\section{The models preparation}

Initially, the model adopted the classic honeycomb structure. Along with the subsequent numerical tests, based on the results obtained, some changes were introduced in the structure. Among other things, the thickness of the cell walls, the dimensions of the basic cell, and the building material of the structure were modified [9].

Based on the literature review the changes to the cells overlap with the contour of the truss were proposed. In the place where the space should be filled with cells, it has a thicker wall. Due to the limitations imposed by the technology additive model can consist of a relatively large empty space. It is therefore proposed to fill in these cells hexagonal. They have for e.g. thinner wall.

Here proposed that the parameters which are prone to change they are wall thickness and cell size. In the case of wall thickness variations in the contour of the grid cell will have a thicker wall than the other. When the cell size will be changed in place of stroke it occurs density smaller cells. In the areas of "empty" cells it will be less, as will be higher. In the first stage it was decided to examine the effect of wall thickness on the obtained results. The dimensions of the model, the approximate dimensions of the tested bone samples were $4 \times 4 \times 40 \mathrm{~mm}$. We adopted this assumption in order to facilitate comparison of the obtained results. Away from the base to the opposite side of the cube was $1 \mathrm{~mm}$. This is an external dimension of a cell. At this stage, we adapted it to the outer dimensions of the model. Changes in internal dimensions - wall thickness - result in a decrease or increase the free space inside the cell. All it correlated to the value of $1 \mathrm{~mm}$.

The wall thickness was $0.4 \mathrm{~mm}$ maximum and a minimum of $0.05 \mathrm{~mm}$. It decided to make changes in different ways. The basic wall thickness is assumed to be 0.25 . The initial model was uniform and the wall thickness of each cell is the same. Initially, the increased wall thick-ness situated on the outline of the grid and at the same time reduces the thickness of the cell walls outside contour. In a next step it was decided that the difference between the thickness and a second cell is not too high. For example, when the contour of the cell wall in the grid has a wall thickness of $0.25 \mathrm{~mm}$, a cell wall beyond the periphery is $0.2 \mathrm{~mm}$ [10].

Based on literature research and the obtained results of simulation of the numerical three-point bending test, changes in the geometry of the sample, and more specifically the empty spaces, were proposed. In the place of a rapid transition between the larger and smaller cells there were stress accumulation, as well as the direction of the crack propagation. They accumulated on combining two layers of cells. Combining it was also another layer of melted ABS material in the FDM 3D printing method. This change consisted of adding a transitional layer between the void layers. Simplifying it, you can compare it to a line connecting alternating cells of different dimensions at the right angle (Fig. 2). This angle depends on how large the difference in dimensions of opposed empty spaces is.

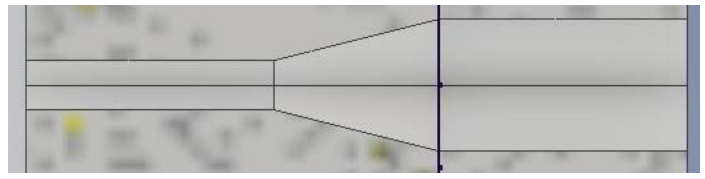

Fig. 2 The example of a transition between cells

The basic model with empty spaces was modelled so that changes in cell size introduced in one cell would automatically take place in the other cells. This significantly facilitated the introduction of changes and significantly reduced the time of modelling subsequent models. Therefore, modelling in the array was used. Due to the specificity of the base structure - honeycomb type - and bandwidth reshaping, it was impossible to model all cells with one pattern. In the final version there are two rectangular constructions in one larger formation. The same principle was adopted for models with a transition. Here, however, you had to watch whether the modelled transitions also change their size. In most cases, the program did not have problems with the command. However, it happened that for some unknown reason the model crumbled. You had to manually change the size of more cells.

Figs. 3 - 8 present a cross-section of models with a larger difference between cell dimensions (Fig. 3, 4, 6, 7) and with a smaller difference between dimensions of opposite cells (Fig. 5, 8).

There were prepared 28 models of which the initial one consisted of one layer. They were made for illustrative purposes. They checked how the structure with the proposed changes - the modeled "voids" - behaves.

The next models had two layers, but they did not have milder transitions between the cells. The next modification was the introduction to the models of transitions between layers of different cells. The last modification concerned the rescaling of models in order to adapt them to the requirements of your 3D printer.

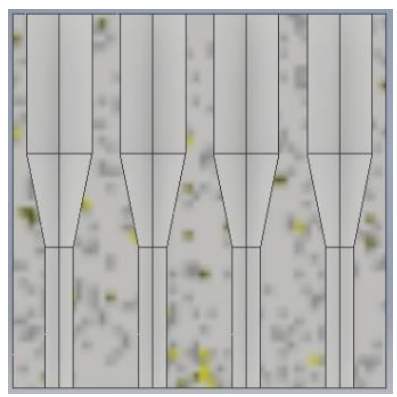

Fig. 3 The cross-section of model with a larger difference between cell dimensions (model 10 a) 


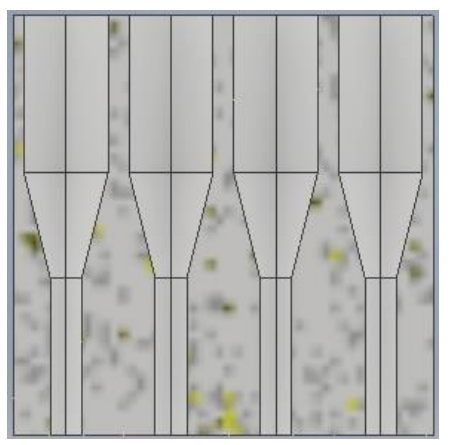

Fig. 4 The cross-section of model with a larger difference between cell dimensions (model 16 a)

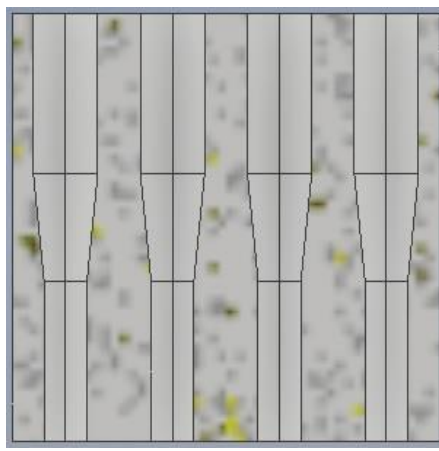

Fig. 5 The cross-section of model with a smaller difference between cell dimensions (model 23 a)

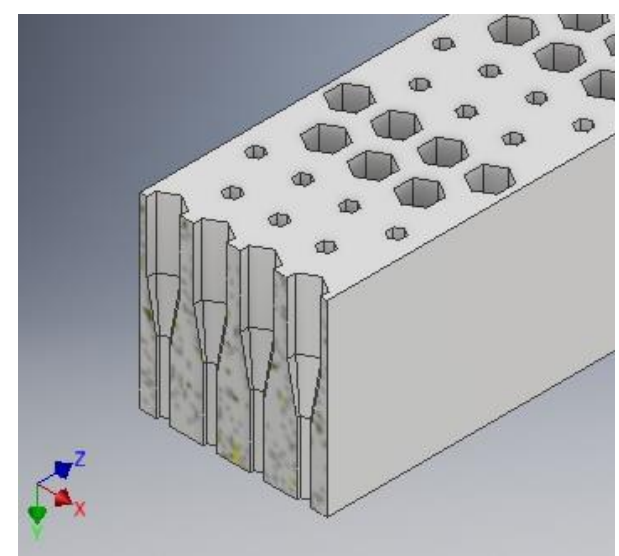

Fig. 6 The section of model with a larger difference between cell dimensions (model 10 a)

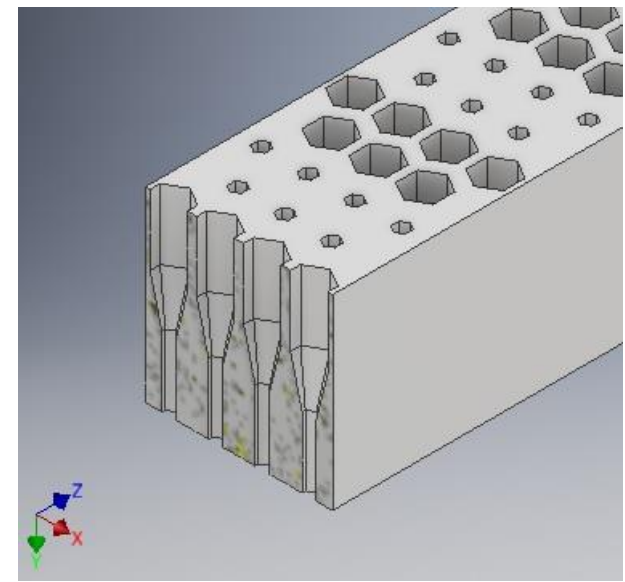

Fig. 7 The section of model with a larger difference between cell dimensions (model 16 a)

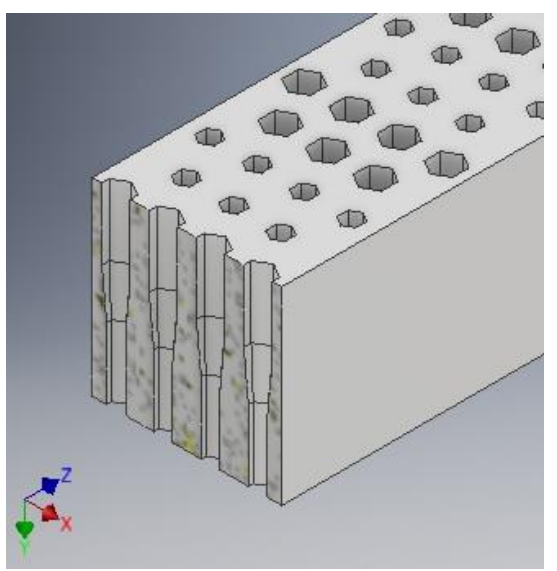

Fig. 8 The section of model with a smaller difference between cell dimensions (model 23 a)

\section{The experimental research}

The experimental study was carried out on a MTS Insight 10 testing machine specially prepared for this study. The stand had to be adapted to the tested samples of small dimensions and forces used. The view of the measuring station - the testing machine is shown in Fig. 7.

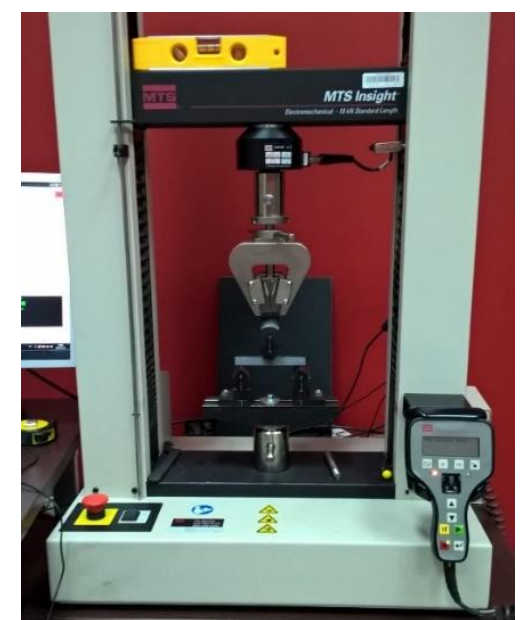

Fig. 7 MTS Insight 10 testing machine prepared for testing

The tests were carried out on samples made with the FDM rapid prototyping technique. Based on previous studies, the dimensions of numerical models were $4 \times 4 \times 40 \mathrm{~mm}$. Therefore, the size of smaller and larger cells had to be small enough. Due to limitations imposed by the manufacturing technique, the samples had to be scaled. They were enlarged three times, which gave analogously $12 \times 12 \times 120 \mathrm{~mm}$ values. The head from standard to smaller has also been changed. Thanks to this change it was possible to create modeled samples on an available 3D printer Prusa i3 MK2. The changes introduced in the numerical model, and due to the phenomenon of notch, were also beneficial from the technological point of view. The printer has managed to produce earlier samples. However, we were not able to check the print quality at critical locations because they were inside the sample. Knowing the capabilities of a printer, we are able to say that it coped better with creating samples with a "transition". An example of a sample created using 3D printing is shown in Fig. 8. In the case of a testing machine, testing on initial, smaller samples would not cause 
major problems. Research on this type of samples has already been carried out before.

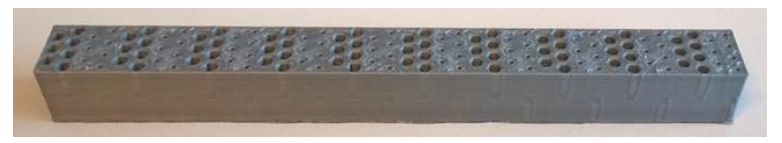

Fig. 8 An example of a sample

Table 1

Selected parameters of the models

\begin{tabular}{|c|c|c|c|c|c|}
\hline Model & $\begin{array}{c}\text { Big cell, } \\
\mathrm{mm}\end{array}$ & $\begin{array}{c}\text { Small cell, } \\
\mathrm{mm}\end{array}$ & Mass, g & $\begin{array}{c}\text { Volume, } \\
\mathrm{cm}^{3}\end{array}$ & $V_{m} / V$ \\
\hline $10 \mathrm{a}$ & 2.1 & 0.9 & 14.384 & 13.570 & 0.785 \\
\hline $16 \mathrm{a}$ & 2.4 & 0.9 & 13.395 & 12.637 & 0.731 \\
\hline $17 \mathrm{a}$ & 2.4 & 1.2 & 12.857 & 12.129 & 0.702 \\
\hline $18 \mathrm{a}$ & 2.4 & 1.5 & 12.192 & 11.502 & 0.666 \\
\hline $22 \mathrm{a}$ & 1.8 & 0.9 & 15.246 & 14.383 & 0.832 \\
\hline 23a & 1.8 & 1.2 & 14.731 & 13.897 & 0.804 \\
\hline 28a & 0 & 0 & 18.317 & 17.28 & 1 \\
\hline
\end{tabular}

$V_{m}$ - volume of sample with voids, $V$ - volume of the full sample

Six series of trials were carried out with five samples in each. They differed in the dimensions of smaller and larger cells. As a result, they had different volume and mass. Volume of empty samples was equated to the same but full sample. The values that were obtained are shown in Table 1. Depending on the type of the sample, there was another crack propagation (Fig. 9, 10).

As a result of the tests carried out on the strength machine, the following average values presented in table 2 were obtained for subsequent samples. Most of the samples did not break. Only in the case of 23 a sample series each was broken. In the case of these samples, the greatest force was observed, the mean displacement being the highest.

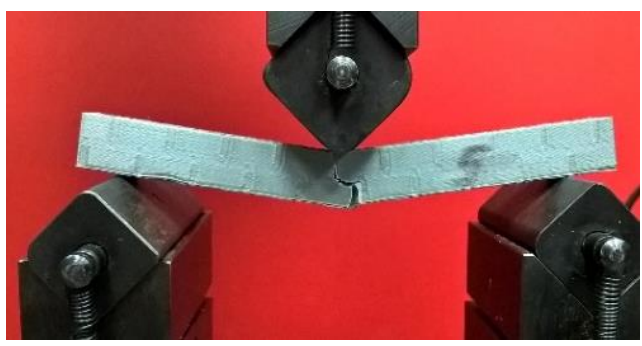

Fig. 9 The crack propagation in sample $16 \mathrm{a}$

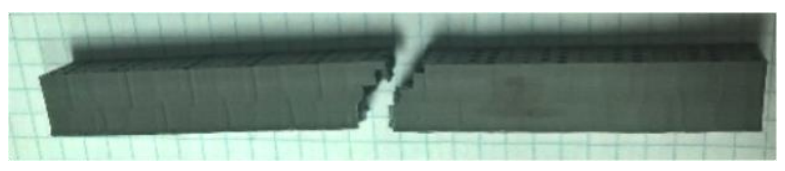

Fig. 10 The crack propagation in sample $23 \mathrm{a}$

Table 2

The average values of load and deflection in experiment

\begin{tabular}{|c|c|c|}
\hline Model & Peak Load, N & Deflection, $\mathrm{mm}$ \\
\hline $10 \mathrm{a}$ & 248.575 & 5.04 \\
\hline $16 \mathrm{a}$ & 151.257 & 3.58 \\
\hline $17 \mathrm{a}$ & 155.093 & 3.78 \\
\hline $18 \mathrm{a}$ & 155.735 & 4.46 \\
\hline $22 \mathrm{a}$ & 288.895 & 4.16 \\
\hline $23 \mathrm{a}$ & 312.047 & 4.92 \\
\hline
\end{tabular}

\section{The numerical simulation}

In the next step numerical simulation of three-point bending test was performed. All numerically tested samples have the same dimensions as a previous tested samples prepared from ABS. The samples with a length of $120 \mathrm{~mm}$, a height of $12 \mathrm{~mm}$ and $12 \mathrm{~mm}$ wide were modeled for each experimentally tested models. The models prepared in CAD system were imported to MSC. Software and before meshing material parameters were assumed. For ABS assumed Young modulus equals $1600 \mathrm{MPa}$ and Poisson's ratio 0.38 [11]. Boundary condition (support and load) assumed as typical during tree-point bending test (Fig. 11). The spacing of supports is $100 \mathrm{~mm}$.

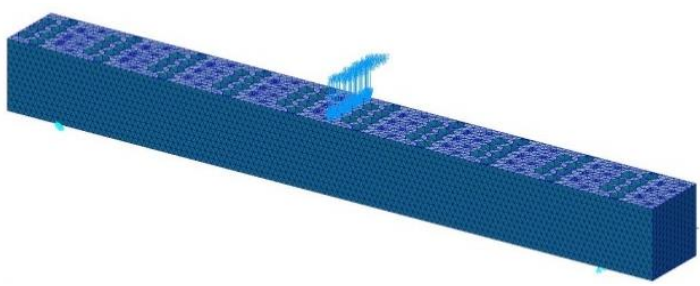

Fig. 11 Boundary condition assumed during numerical simulation

The acting force assumed as maximal force for each test respectively (Table 2). The selected results for models 10 a, 16 a and 23 a are presented in Figs. 12-20.

Discretization of the models was carried out with tetrahedral elements of the Tet 4 type with a linear shape function. The average distance between nodes was taken equal to $1 \mathrm{~mm}$. The developed models are composed of approximately 400,000 elements and have approximately 120,000 degrees of freedom.

Vertical displacements $(\mathrm{Y})$, reduced stresses (von Mises) and normal stresses along the longitudinal axis of the sample (Z) were selected as representative results. There is only one picture presented vertical displacement (for 10a model) because the displacement distribution for all models is the same - the difference appears in values.

The distribution of normal stresses presented in Figs. 19 and 20 (for the cross-section with the highest values of tensile and compressive stresses, respectively) well illustrates the mechanism of fracturing the samples observed in the experiment. In samples, a break (gap) always appears from the bottom in the section where the loading force (or in close proximity) is acting, where the largest holes are located. In the vast majority of cases, the fracture does not pass through and with a sharp drop in force and a further increase in deflection, the upper compression zone is still not damaged and transfers loads.

Although the obtained distributions and values of both reduced and normal stresses confirm the results obtained in experimental studies, the displacement values obtained in the numerical tests are slightly smaller (about $20 \%$ ) than in the experiment. Probably the Young modulus based on the literature data does not correspond to the actual value obtained after the samples were made by incremental $3 \mathrm{D}$ printing. This issue requires further research and the socalled "tuning" of the model. 


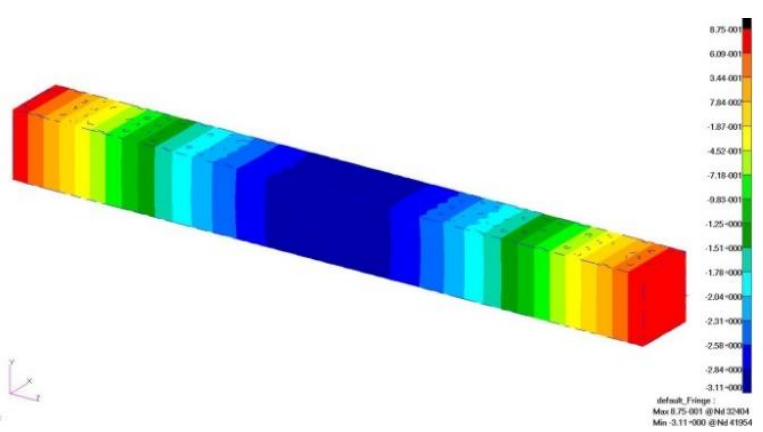

Fig. 12 Vertical displacement Y (in mm) for 10 a model

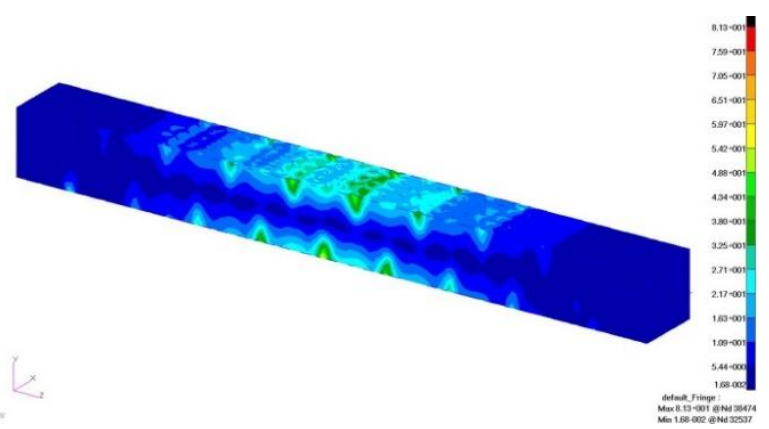

Fig. 13 The reduced stresses (von Mises, in MPa) for 10 a model

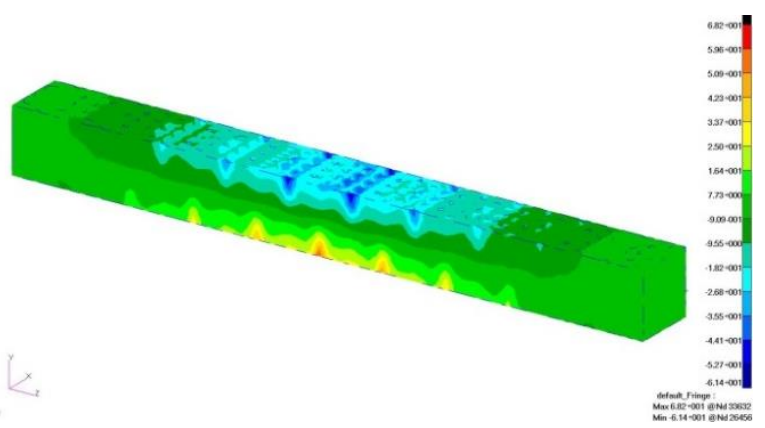

Fig. 14 The normal stresses (in MPa) for 10 a model

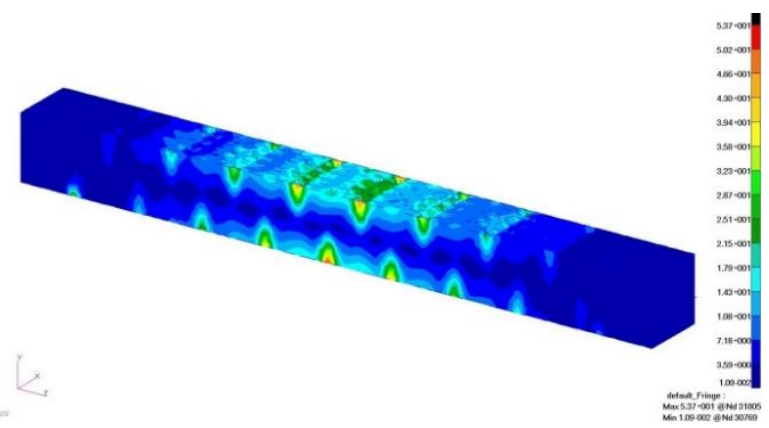

Fig. 15 The reduced stresses (von Mises, in MPa) for 16 a model

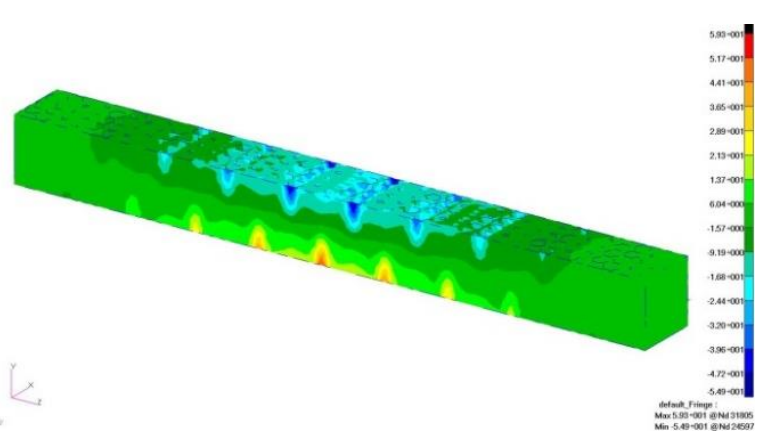

Fig. 16 The normal stresses (in MPa) for 16 a model

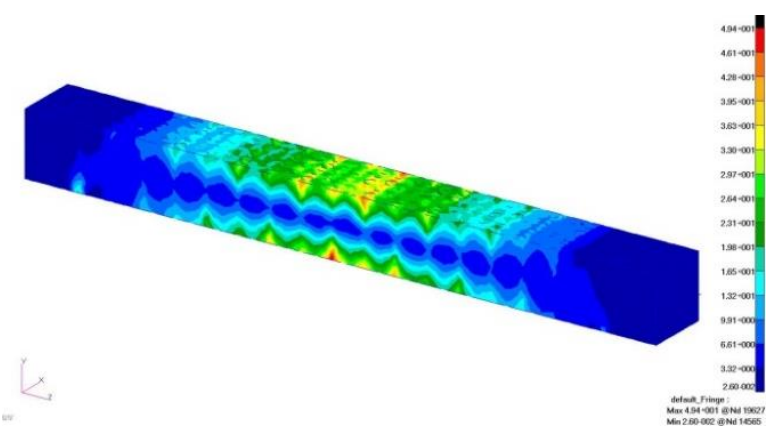

Fig. 17 The reduced stresses (von Mises, in MPa) for 23 a model

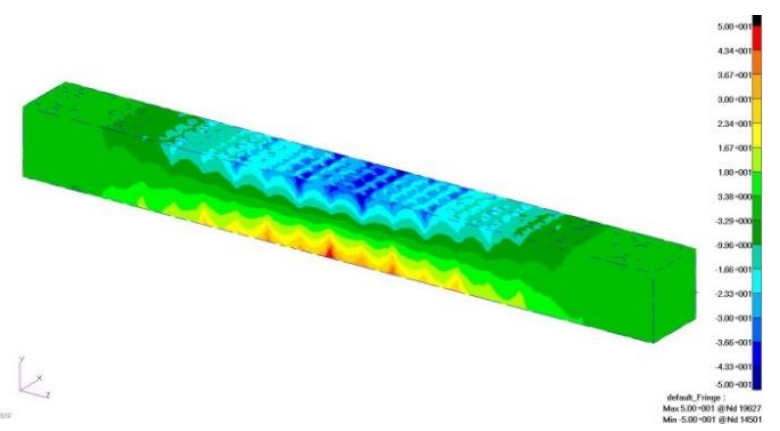

Fig. 18 The normal stresses (in MPa) for 23 a model

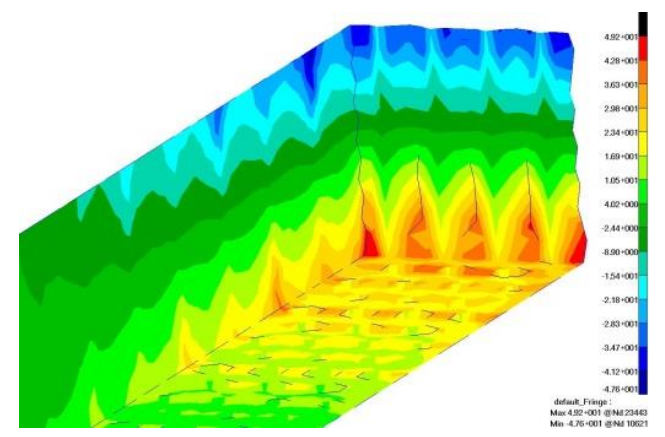

Fig. 19 The normal stresses (in MPa) for 23 a model, crosssection with the highest tensile stresses

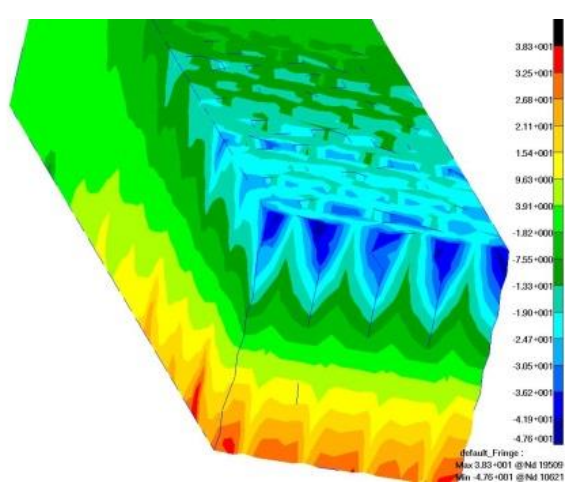

Fig. 20 The normal stresses (in MPa) for 23 a model, crosssection with the highest compression stresses

\section{Conclusions}

The research carried out was aimed at developing a lightweight structure and at the same time durable. An additional important limitation is the possibility of creating a structure in incremental 3D printing technology.

The results obtained from numerical simulation well illustrates the mechanism of fracturing the samples ob- 
served in the experiment. However, the obtained distributions and values of stresses confirm the results obtained in experimental studies the deflection obtained in the numerical tests are smaller than in the experiment. It is observed that during manufacturing process the material properties change and may differ from those given for filament. This requires further research and "tuning" of the model.

\section{References}

1. Wahl, L.; Maas, S.; Waldman, D. 2011. Shear Stresses in Honeycomb Sandwich Plates: Analytical Solution, FEM, and Experimental Verefication; University of Luxrmbourg.

2. Abbadi, A.; Koutsawa, Y.; Carmasol, A.; Belouettar, S.; Azari, Z. 2009. Experimental and numerical characterization of honeycomb sandwich composite panels, Simulation Modelling Practice and Theory 17: 15331547. https://doi.org/10.1016/j.simpat.2009.05.008.

3. He, M.; Hu, W. 2008. A study 2 on composite honeycomb sandwich panel structure, Materials and Design 29: 709-713. https://doi.org/10.1016/j.matdes.2007.03.003.

4. Fengnian, J.; Hailong, C.; Long, Z.; Hualin, F.; Chuanguo, C.; Ning, K. 2013. Failure mechanisms of sandwich composites with orthotropic integrated woven corrugated cores, Experiments, Composite Structures 98: 53-58. https://10.1016/j.compstruct.2012.09.056.

5. Neimitz, A. 1998. Fracture mechanics, Wydawnictwo Naukowe PWN, Warszawa (in Polish).

6. Butnicki, S. 1979. Weldability and brittleness of steel. Wydawnictwa Naukowo-Techniczne, Warszawa (in Polish).

7. German, J. 2011. Basics of fracture mechanics, Kraków 13-22 (in Polish).

8. Kosior-Kazberug, M.; Kazberug, A. 2015. The influence of notch rounding in the test piece on parameters of fracture mechanics of cementitious composite, Ceramic materials 67: 413-417 (in Polish).

9. John, A.; John, M. 2016. Foam metal and honeycomb structures in numerical simulation, ANNALS of Faculty
Engineering Hunedoara - International Journal of Engineering, T. XIV, No. 4 [Nov.], 27-32.

10. John, M.; John, A.; Skarka, W. 2017. The influence of the geometrical parameters on the mechanical properties of honeycomb structure, Mechanika 23(3): 359-364. http://dx.doi.org/10.5755/j01.mech.23.3.18478.

11. ABS. Technical data. Ultimaker. [accessed 20 May 2017]. Available from Internet (in Polish): https://ultimaker.com/download/67637/TDS\%20ABS\%20v3.011-pol\%281\%29.pdf

\section{John, A. John, W. Skarka}

\section{THE INVERSE HONEYCOMB STRUCTURES IN NUMERICAL MODELING AND EXPERIMENT}

\section{S u m m a r y}

Based on previous research, we proposed changes in the classic honeycomb structure. We have changed the approach to modeling. In a classic structure, cells are modeled using the walls that form them. In the modified structure, the main modeled element is the hexagon-shaped void. The distribution of cells was proposed as in a truss. In full places the spaces were smaller and in empty places the cells were larger. We changed the size of cells and the transition between cells in different layers.

In comparison to previous research, it was necessary to enlarge the models. This was due to the limitations of incremental technology, which was used to fabricate samples - FDM. This technology introduced limitations of changes that the structure underwent. We selected several types of models as a result of the numerical simulation and produced. Then, experimental tests were carried out on them - the same as in numerical simulation. In the final stage, we compared the obtained results.

Keywords: invers honeycomb structures, 3D printing, FDM, tree point bending test, numerical simulation.

Received April 20, 2018 Accepted June 14, 2018 